\title{
Optimal climate policy and the future of world economic development
}

\author{
Mark Budolfson Francis Dennig Marc Fleurbaey \\ Noah Scovronick Asher Siebert Dean Spears* Fabian Wagner
}

April 10, 2017

\begin{abstract}
How much should present generations sacrifice to reduce emissions today, in order to reduce the future harms of climate change? Within climate economics, debate on this question has been focused on so-called "ethical parameters" of social time preference and inequality aversion. We show that optimal climate policy similarly importantly depends on the future of the developing world. In particular, although global poverty is falling and the economic lives of the poor are improving worldwide, leading models of climate economics may be too optimistic about two central predictions: future population growth in poor countries, and future convergence in total factor productivity (TFP). We report results of small modifications to a standard model: under plausible scenarios for high future population growth (especially in sub-Saharan Africa) and for low future TFP convergence, we find that optimal near-term carbon taxes could be substantially larger.
\end{abstract}

\footnotetext{
${ }^{*}$ Budolfson: Woodrow Wilson School and University Center for Human Values, Princeton University, Princeton, NJ 08540. Dennig: Social Science (Economics), Yale-NUS College, Singapore, 138610. Fleurbaey: Woodrow Wilson School and University Center for Human Values, Princeton University, Princeton, NJ 08540. Scovronick: Woodrow Wilson School, Princeton University, Princeton, NJ 08540. Siebert: International Research Institute for Climate and Society, Earth Institute, Columbia University, New York, NY. Spears (corresponding author): Economics and Planning Unit, Indian Statistical Institute - Delhi Centre, Delhi, India; Woodrow Wilson School, Princeton University, Princeton, NJ 08540. dspears@princeton.edu. Wagner: Woodrow Wilson School and Andlinger Center for Energy and the Environment, Princeton University; International Institute for Applied Systems Analysis (IIASA), Laxenburg, Austria. We appreciate comments from participants at NEUDC, the Eastern Economic Association, the Population Association of America annual meeting, and Princeton University; errors are our own.
} 


\section{Introduction}

How much should present generations sacrifice to reduce emissions today, in order to reduce the future harms of climate change? Economists answer this question using Integrated Assessment Models of optimal carbon taxes, which balance near-term costs against future climate damages (Pizer et al., 2014). Within climate economics, debate on this question has been largely focused on so-called "ethical parameters" of social time preference and inequality aversion. Although there is growing recognition of the importance of the climate vulnerability of the future poor (Hallegatte et al., 2015), leading models of climate economics make assumptions that do not reflect the full range of plausible future scenarios for economic and human development. It is therefore a priority for climate economics and policy to know how sensitive optimal carbon taxes are to other likely futures for the world's poor. We show that optimal climate policy importantly depends on the future of development, with effects that are comparable in size to large changes in ethical choices such as discounting.

The 2016 report of the U.S. National Academies on the social cost of carbon highlighted that better socioeconomic models and projections are a critically needed input into assessing climate policies (National Academies, 2017). In this paper, we investigate how important the contributions of development economics, in particular, may be to efforts to construct

policy-relevant models of climate change and emissions policies: how much does optimal climate policy depend upon the answers to development economists' questions? We study the effects on optimal climate policy of two types of assumptions about the future of the developing world: different possibilities for population growth and its distribution worldwide, different possibilities for future convergence in aggregate productivity, and plausible interactions among these possibilities. The results make three contributions to the literature on optimal climate policy.

First, this paper theoretically emphasizes that population growth can play a role analo- 
gous to time discounting in determining optimal climate policy; this is because a larger future population increases the social importance of the future, relative to the present. However, despite this importance, population has not received the same systematic or consistent treatment as other aspects of the social welfare function, such as time preference or inequality aversion.

Second, this paper quantifies the effects on the optimal tax of population assumptions. Largely because of slow fertility decline in sub-Saharan Africa, the most recent global population projections now project that world population growth is likely to remain positive at the end of the 21st century (Gerland et al., 2014). We document a mechanism for the effect of population assumptions that changes over time: in the near term, greater future population growth increases optimal taxes because of its role in the social welfare function; in the long term, a larger realized population matters because of its effects on the economy and emissions. Future population growth influences optimal present day Pigouvian taxes under a social welfare function that regards externality costs to more future people as a greater social cost. Under the UN's "low" projected future population path, the optimal global temperature is about $0.75{ }^{\circ} \mathrm{C}$ greater than under the UN's "high" projected future population path; this is because a given level of climate damages registers as a greater social cost when more people will suffer from it.

Third, this paper computes optimal carbon taxes under assumptions of slower and faster convergence in Total Factor Productivity between the developing and developed world than is assumed by some leading models of optimal climate policy. Understanding the determinants of aggregate productivity is an active open research question in development economics (Easterly and Levine, 2001). Indeed, historical literature on economic growth contains decades of predictions of macroeconomic convergence; however, evidence indicates that country-level income in fact has diverged (Pritchett, 1997), or has failed to converge (Grier and Grier, 2007), or has perhaps converged at best only very slowly (Deaton, 2013). Lowering the as- 
sumed rate of convergence of poor countries' TFP growth to rich countries' has two effects: in the near term, it raises optimal carbon taxes because climate damages are hurting a future developing world that is poorer than it otherwise would be; in the long term it lowers optimal taxes because the world can afford to pay less. We show that assumptions about population growth and TFP interact: if the future developing world were expected to have a large population and low TFP, then today's optimal carbon tax would increase considerably.

In many ways, life is getting better quickly for people in poor countries: infant mortality is falling, the fraction of people who are very poor is falling, populations are getting taller on average. But there are at least two important exceptions to these improving trajectory: one, that the fertility transition in sub-Saharan Africa is not following the same pattern as in other regions of the developing world, resulting in persistent positive population growth; and, two, that despite decreasing inequality among individuals at the world level, aggregate economic convergence has been slow or absent, perhaps especially in TFP. The causes of these two facts are not fully agreed upon in their respective literatures; as a result, successive expert projections have proven subject to large revisions. These two facts matter here because among the many positive and negative trends in the developing world - it turns out that these two trajectories are important determinants of optimal carbon taxes, as computed by economists' conventional models.

These findings are important for climate policy. Much of economists' debate about climate policy has considered the "ethical parameters" of time discounting and inequality aversion (Arrow et al., 2013). However, the future population and economic growth of the developing world are similarly important for optimal carbon taxes; are amenable to scientific refinement (through improved demographic projections, growth theory, and econometrics); and may be treated over-optimistically in the assumptions of influential models. Therefore, this paper advances a growing literature that documents the importance of the vulnerability of developing countries to climate change in the future (World Bank, 2010), above and 
beyond the increasingly well documented high levels of exposure of people in developing countries to environmental pollution today (e.g.Kumar and Foster, 2007; Tanaka, 2015). Our results underscore the inseparability of climate economics from development economics (Greenstone and Jack, 2015).

\section{Background: IAMs, population, and TFP growth}

How much should society today be willing to sacrifice in order to prevent future climate damages? Under Executive Order 12866, U.S. government agencies have been required to incorporate into regulatory decisions a "social cost of carbon," computed from economic Integrated Assessment Models (IAM). Such models incorporate economic optimization with scientific models of the climate and economic models of growth, in order to inform regulation and, in some models, compute a socially optimal tax on carbon, sometimes called a "carbon price" (Kossoy et al., 2015). In particular, IAMs weigh future climate damages against present-day mitigation costs to investigate optimal climate mitigation policy.

Debates over the social discount rate have proven important in economic valuations of climate change because most of the damage will happen decades or centuries in the future; a social planner that is less concerned about future well-being will be willing to incur lower costs today to prevent climate damages. It is well known that a lower social discount rate would recommend a greater reduction in carbon emissions. We introduce optimizing IAMs in order to show that assumptions about the future of the developing world can be of importance similar to choice of a social discount rate.

Any economic model to estimate an optimal tax ${ }^{1}$ requires a social welfare function (SWF) in order to value the tradeoffs society faces in its policy choices. For example, two forms of

\footnotetext{
${ }^{1}$ Note that the optimal tax does not appear directly in equation 2.1, as it is neither subtracted from consumption, nor redistributed to the population (Mankiw, 2009); because our model, like other IAMs, uses a single representative agent within each region, carbon taxes are simply consumed like other output that is not invested as savings. Instead, a carbon tax indirectly reduces consumption by reducing economic output.
} 
social welfare function that have been discussed in the population literature are average utilitarianism, which averages well-being over members of a population, and total utilitarianism, which adds well-being over members of a population, and therefore considers additional good lives to be an improvement in social welfare. Generically, all three cost-benefit optimization IAMs use the same default social welfare function of a discounted total utilitarian form, as described by Pindyck (2013):

$$
W=\frac{1}{1-\eta} \mathbb{E}\left[\int_{2015}^{2300} L_{t} C_{t}^{1-\eta} e^{-\rho t} d t\right],
$$

where $\mathbb{E}$ indicates an expectation over possible futures, $t$ indexes time, $\rho$ is the rate of social pure time preference, and $\eta$ is a parametrization of the curvature of the within-period felicity function of period-average consumption $C_{t}{ }_{2}^{2} L_{t}$ is population size in period $t$.

If the social discount rate $\rho$ is positive, costs to future generations are discounted in the computation of optimal present-day tax because these costs accrue to people living further in the future. As many observers have commented, much of the difference between the relatively high recommended carbon taxes of Stern's (2006) IAM and the relatively low carbon taxes of Nordhaus' RICE IAM can be accounted for by the fact that RICE assumes a larger positive social discount rate, although this is not the only difference between these models and approaches. RICE, the model that we build upon in our analysis, is introduced in the next subsection.

\subsection{Introducing the RICE model}

All analyses in this paper use the Regionalized Integrated Climate Economy (RICE) model (Nordhaus and Boyer, 2000). The RICE/DICE model family (DICE being the global coun-

\footnotetext{
${ }^{2}$ Alternative models have relaxed some of these constraints. Strictly, Pindyck (2013), in his equation 1 , omits $L$, implicitly assuming within-period average utilitarianism. Nordhaus (2011), in his equation 1 , incorporates $C$ and $L$ as arguments into a within-period function $U$; although this is not discussed in the text, the only purpose for this flexibility could be to allow curvature over population size.
} 
terpart) is one of three leading cost-benefit climate economy models capable of estimating the social cost of carbon (PAGE and FUND are two others). RICE is highly representative of the set of leading cost-benefit IAMs that are used to estimate the social cost of carbon, so the results we find with RICE should not be qualitatively different if investigated with any of these models.

First developed in the 1990s, and updated several times subsequently, the RICE model consists of 12-regions, some of which are large countries and some of which are sets of countries. Each region has its own "damage function," an equation that translates the temperature change due to climate change into the damages that that region eventually experiences. The DICE/RICE models are freely available and have been used extensively in both academic research and policy analysis, including in the US governments Interagency Working Group on The Social Cost of Carbon, which recommends a carbon price for use in legally mandated cost-benefit analyses that evaluate relevant regulations. RICE has been described in detail elsewhere, but we also provide a detailed specification in the online supplementary appendix.

\section{$2.2 \quad$ Population growth}

Recent estimates by the UN World Population Prospects find that there is an important probability that population will be as high as 12.3 billion in 2100 (Gerland et al., 2014). These projections estimate that there is a $70 \%$ chance that population growth will still be positive in 2100. In particular, the median projection for Africa in 2100 is 4.2 billion people 3 and still growing, compared with less than 1 billion today. Population growth in Africa may be especially important for the social cost of carbon in regionally disaggregated models if climate damages are expected to be large there, or if economic evaluations of social welfare are sensitive to inequality or to the likelihood that poor Africans would be disproportionately

\footnotetext{
${ }^{3}$ Which would give Africa a population density comparable to China today.
} 
harmed.

In this section, we introduce the importance of population projections to IAMs, and in particular the importance of population to some SWFs.4 First, we note that the set of possible future population paths in the literature contains a wide diversity of possible futures; over the past decades, demographers' best predictions about future population growth have been repeatedly revised under new information, and there is no reason to be sure that large revisions could not happen again. Second, we note that under the SWF used in leading IAMs, population growth plays a role formally analogous to time discounting. Third, we note that among SWFs used in the literature, there is indecision or implicit disagreement about how future population should be incorporated into climate policy. Our work on population in this paper builds on a shorter paper by the same authors in the climate science literature (Scovronick et al., 2017), which considers only the global population total, and is therefore unable to highlight the importance of the future of the developing world.

\subsubsection{Population growth: assumptions and projections}

Possibilities for the future of human population size vary widely. Panel (a) of figure 1 presents four population paths that we will use throughout this paper. The high, medium, and low paths are the most recent UN aggregate population projections, taken from the 2015 round of the World Population Prospects. Note that in the medium projection, world population growth is still positive in 2100, largely because of positive population growth in Africa, as shown in panel (b).

The fourth projection is the population projection used in Nordhaus $s$ (2011) implementation of RICE. Since that time, a newer version of RICE has slightly updated its population assumptions to more closely match the newest UN projections. Therefore, we emphasize that

\footnotetext{
${ }^{4}$ Our purpose is to consider consequences of whatever exogenous population growth may be; we do not consider policy options to change population growth, or what an optimal population policy would be (Spears, 2015 ).
} 
we do not use these figures to argue that RICE is "wrong." Rather, we include the comparison between the 2011 RICE assumptions and the most recent UN projection throughout our analyses in order to document the quantitative importance for optimal taxes of a modest change in population assumptions that actually occurred in the recent history of climate modeling.

Much of the uncertainty in future population size is from developing countries, especially from Africa, but also importantly from Asia (Gerland et al., 2014). This is largely because fertility-decline in sub-Saharan Africa has lagged considerably behind the fertility transition in other developing regions, for reasons that are not fully understood even by frontier demographic research $($ Kohler, 2012$)$. Reviews of past population projections show that even the most authoritative past projections have proven importantly incorrect, or have subsequently been considerably revised (Lam, 2011). In light of this history, and of the considerable uncertainty in even the best demographic literature, it is plausible that future revisions in population projections could reflect differences as large or larger than the difference we study, between the 2011 RICE assumptions and the 2015 WPP projections.

We note that one important possibility is that climate change could endogenously influence world populations through net migration. Our population paths consider migration in the sense that the exogenously assumed WPP projections themselves include migration assumptions. However, in this paper we abstract away from any endogenous effect of climate change on net migration, to avoid further deviation from RICE $i$ 


\subsubsection{Population growth can be theoretically analogous to time discounting}

For each time period $t$, define $g_{t}$ as the population growth rate that correctly makes $L_{t}=$ $e^{g^{t} t} L_{2015}$. Then the generic SWF of equation 2.1 can be rewritten:

$$
\begin{aligned}
W & =\frac{1}{1-\eta} \mathbb{E}\left[\int_{2015}^{2300}\left(e^{g_{t} t} L_{2015}\right) C_{t}^{1-\eta} e^{-\rho t} d t\right] \\
& =\frac{L_{2015}}{1-\eta} \mathbb{E}\left[\int_{2015}^{2300} C_{t}^{1-\eta} e^{\left(g_{t}-\rho\right) t} d t\right] .
\end{aligned}
$$

This functional form emphasizes that the weight of the utility of a period's average consumption in the social welfare function depends on both the population growth rate and the time discount rate. If population growth is high enough relative to the discount rate, then average consumption in future periods is more important than average consumption in the present: 5

As equation 2.2 shows, different population paths are important because they reweight the distribution over the total human population of economic costs and benefits. This reweighting happens over two dimensions: time and space.

The importance of the time dimension of population reweighting is visible in figure 2. Figure 2 illustrates this point by presenting optimal mitigation policies computed by RICE, under the idealized assumption of constant global population growth rates. An increase in the constant, worldwide population growth rate of 0.1 percentage points results in an approximately 7 percent higher near-term optimal tax ${ }^{6}$ This occurs because higher population growth means that the damages suffered by a (larger) future population are weighted

\footnotetext{
${ }^{5}$ In fact, not all studies of optimizing climate IAMs are explicit that they use a SWF of this totalpopulation type; some papers do not describe an unambiguous assumption about population, because the economic or scientific focus of the research is on a different dimension of climate policy. Pindyck (2013), for example, omits population from his SWF, implicitly appearing to assume discounted within-period average utilitarianism. Anthoff and Tol (2010), in their IAM named FUND, use a population weighted SWF effectively identical to our equation 2.1. The results of this paper's analysis suggest that it could be useful for the IAM community to systemize assumptions on the role of population in social welfare.

${ }^{6}$ We compute this semi-elasticity by regressing the log of optimal taxes in 2 on the constant population growth rate; $R^{2}$ is greater than $99 \%$.
} 
more heavily in the social welfare function. This reweighting is precisely analogous to time discounting. In equation 2.2, a constant population growth rate would be represented by $g$ without a time subscript, so the passage of time would be multiplied by the constant $g-\rho$ : the difference between population growth, which makes the future more important, and time discounting, which makes the future less important. An equal percentage point increase in constant population growth and time discounting would exactly offset one another.

Population's role in reweighting across world regions is visible in panel (b) of figure 1. This figure decomposes the differences across the UN population projections into the differences contributed by each of the 12 RICE regions. The large central section is sub-Saharan Africa (hereafter referred to as "Africa"), indicating that the population path that the world takes could not only differ substantially in the total numbers of people at different times; it could also differ considerably in the fraction of the world population at any one time that is in Africa. If the world follows a population path such that African population growth remains higher than population growth in the rest of the world for many decades, then the fraction of the population that lives in Africa could increase markedly. Whether this is projected to occur will have a large effect on optimal climate mitigation policy because Africa is a climate vulnerable region: larger mitigation costs are justified today if many more future people in Africa will be exposed to climate damages, all else equal.

\subsection{Economic growth: How quickly will TFP converge globally?}

The RICE IAM is a model of 12 global regions, some of which are large countries and some of which are sets of countries. Figure 3 presents the contemporaneous across-region inequality at each time period, computed from consumption levels in optimal paths for carbon emissions and carbon taxes. The figure plots several lines - each corresponding to a different combination of the "ethical parameters" for inequality aversion and social time preference - but differences among the lines are not visible as plotted. Rather, what the 
graph shows clearly is a strong downward trend in inequality over time, across all modeled assumptions of the ethical parameters. In other words, the RICE model makes the strong assumption that global inequality will certainly fall dramatically over time.

However, some macroeconomists see evidence for a "disadvantage of poverty" (Ravallion, 2012), which could counteract forces of convergence and cause it not to be the case that countries with high levels of poverty grow faster (López et al., 2015). Whether or not overall global inequality is increasing or decreasing depends on whether one looks at country aggregates or attempts to look at person-level inequality, and on whether or not country averages are weighted by population (Milanovic, 2011): although person-weighted measures are dominated by the large, growing economies of China and India, it is clear that inequality of country level aggregates (of the sort that climate IAMs model) has been non-decreasing over time or increasing.

Among the many potential determinants of economic growth, RICE particularly assumes convergence in GDP because the model assumes rapid convergence across regions in Total Factor Productivity (TFP). TFP, famously described by Moses Abramowitz as a "measure of our ignorance," is essentially a residual in growth accounting, the heterogeneity in longrun economic performance that is not accounted for by differences in factor inputs through the functional form of a growth theory. Much remains unknown about what TFP in fact measures, and why it varies so importantly across countries (Hsieh and Klenow, 2010). Technology and productivity differences may be highly persistent over centuries (Comin et al. 2010). As Grier and Grier (2007) show, other inputs such as physical and human capital are converging across countries, so divergence in outcomes appears particularly incompatible with strong convergence in a TFP-type residual. Although this does not mean it could not be useful to consider optimal climate policy in a world of rapid TFP convergence, the growth accounting literature is far from suggesting that such convergence is implied by the facts Caselli, 2005). 
The Penn World Tables quantify TFP in each country over time (Feenstra et al., 2015). These data give little reason to be confident that TFP will converge towards the U.S. in the future. In the past decade, TFP in India and China, relative to the U.S., has hardly increased at all 7 Over the full range of data in the Penn World Tables, TFP in Africa has decreased relative to TFP in the U.S.8 These data therefore suggest to us investigating a case in which TFP does not converge, as well as a case of TFP convergence only half as quickly as in the RICE model.

As in the case of population assumptions, it is not our intent to criticize the RICE IAM or any other. Instead, our goal is to employ the framework of RICE to investigate the sensitivity of optimal climate policy to assumptions about the future of the developing world. It is plausible that developing countries, in the future, will not experience the dramatic catch-up that figure 3 documents to be assumed by some accounts of climate policy; we investigate the implications of this possibility by varying the assumed rate of TFP convergence. Combining different assumptions about TFP convergence with different assumptions about population growth allows us to investigate the plausible case where the future developing world contains many more people than today, who will be exposed to climate change while living in economies that continue to lag considerably behind the richest.

\section{Method: Small modifications of a leading, publicly available IAM}

We produce new estimates of the social cost of carbon by making small modifications to the quantitative assumptions of RICE, a leading regionally-disaggregated IAM, developed by

\footnotetext{
${ }^{7}$ For example, TFP in China (relative to the U.S.) is 0.42 in 2007 and 0.43 in the most recent observation.

${ }^{8}$ In a regression of the TFP level at current purchasing power parity (so the U.S. is always 1 ) on the year, with country fixed effects, for all available African country years, an additional year in the future is, on average, linearly associated with the TFP ratio being 0.0055 lower, with a standard error of 0.0018 , clustered by country.
} 
Nordhaus (2011). 9 In particular, we vary two dimensions of the future of economic development: population growth ${ }^{10}$ and TFP convergence ${ }^{11}$ In the IAM literature, much attention has been paid to the "ethical parameters" of pure time discounting $(\rho)$ and inequality aversion $(\eta)$; because our objective is to make minimal modifications to RICE, in our computations throughout the paper we maintain the RICE assumptions of $\rho=1.5 \%$ and $\eta=1.5$.

\subsection{Alternative population assumptions}

As introduced in section 2.2 and figure 1, we consider four future population paths: the assumptions of Nordhaus's (2011) RICE and the high, medium, and low assumptions of the 2015 UN World Population Prospects (WPP). Although panel (a) plots the time path of

\footnotetext{
${ }^{9}$ Our model is a Matlab implementation of the RICE2010 spreadsheet written for Dennig $(2014)$. The original spreadsheet, by William Nordhaus, can be found here: http://www.econ.yale.edu/ nordhaus/ homepage/documents/RICE_042510.xlsm

In the online supplementary appendix, we present equations that describe our model. Our model replicates all aspects of the original spreadsheet except for (i) the objective function, (ii) the savings rate specification, and (iii) the implementation of the sea-level-rise damages. We use a population weighted discounted utilitarian objective, while the original RICE2010 model uses Negishi-weights in addition to population weights. We use a fixed (Solow) savings rate, while RICE2010 uses endogenous savings rates. Finally, we simplify the sea-level-rise damages by incorporating them into the quadratic damage function coefficients, while in RICE2010 damages consist of a quadratic non-sea-level-rise component and a separate sea-level-rise component. The differences in savings rate and damage specifications are also described in full detail in the section "Modifications to RICE2010" in Dennig et al. (2015).

Our model is additionally similar to NICE, a related IAM by Dennig et al. (2015) which was designed to extend RICE to within-region inequality; in the production of every estimate in this paper, our models are set to ignore within-region inequality.

${ }^{10}$ Our paper builds upon our prior work about aggregate global population in the climate science literature in Scovronick et al. (2017), a study which uses the globally representative model DICE, rather than the regionally disaggregated RICE, and therefore is unable to address the topics at the core of our paper: economic development, population growth in the developing world, the possible co-incidence of population growth with poverty and climate damages across regions, and TFP convergence. Unlike the present paper, Scovronick, et al. specifically study the policy implications for optimal climate mitigation of population and fertility policies and programs.

${ }^{11}$ A related recent paper by Gillingham et al. (2015) also studies variation in IAM output as a result of quantitatively differing inputs. Although the Gillingham study is an important contribution to the literature, our contribution differs in three ways: (1) we focus on uncertainty about the future of development, while in contrast they use the DICE model that studies the world as a single representative-economy global whole; (2) we study policy implications of focal cases of demographers' population projections, while they randomly perturb a central growth rate in order to produce a statistical distribution of results; and (3) a central focus of their paper is quantitatively assessing discrepancies across models, while we focus on understanding the economic role of assumptions, such as in the effect of population growth on the SWF, or the implications of TFP assumptions for future inequality.
} 
global total population, in fact the regional composition of population additionally varies considerably across the paths, as seen in panel (b). The 12 RICE regions are not identical to the UN regions for which the WPP are produced. Therefore, we aggregate countrylevel WPP population projections into the RICE regions. This method reproduces the global population exactly through 2100, the end of the projection horizon. In the weighted average, growth rates are weighted by the starting population of each country. These growth rates are applied forward to observed 2015 population levels to compute projected future population levels. Despite using this different regional aggregation, we find that our 2100 total population levels produced by this method closely match the 2100 population levels produced by WPP.

WPP projections end at 2100, but RICE extends to 2300. We project regional growth rates from 2100 to 2200 by mechanically reducing the 2100 growth rate linearly to zero in

ten steps, thus the 2110 growth rate is 0.9 times the 2100 growth rate, and so on. We assume zero population growth in all WPP cases from 2200 onwards. Note that the RICE population path assumes a slight decline in world population from 2100 to 2200 followed by a slight increase from 2200 to 2300. By the end of the period studied, the difference between the RICE and WPP medium population paths becomes large: 8.7 versus 11.4 billion people.

\subsection{Alternative TFP convergence assumptions}

RICE assumes that TFP converges at a rate of 0.1 per decade, meaning that as each decade passes, the gap between a developing region's TFP and the asymptotic value of that region's TFP reduces by 10 percent (for details, see the appendix). This assumption may be strong in light of a literature in development economics in which TFP is imperfectly understood and not certainly converging. We consider four TFP convergence rates: 0 (no convergence), 
0.05 (half of RICE convergence), 0.1 (RICE), and 0.2 (convergence twice as fast as RICE).$^{12}$ We first consider the implications of these assumptions separately at the medium population path and then interact TFP assumptions with alternative population assumptions.

\section{Optimal tax and population projections}

\subsection{Result: Population growth and time discounting}

Figure 4 presents the main result of this section: optimal carbon taxes are considerably higher when high population growth paths are assumed rather than low population growth paths. As the difference between the RICE-2011 and UN WPP-2015 medium tax trajectories shows, even a relatively modest increase in projected population, of the magnitude that has in fact occurred in recent demographic updates, is sufficient to visibly increase optimal taxes: the optimal 2025 carbon tax is $25 \%$ greater under the revised population assumption. This update also moves closer in time by several decades the point at which the economy is projected to hit the "backstop" technology: exogenous future technological progress is assumed to make full decarbonization optimal once carbon taxes become large enough, which is visible in the graph as the common downward trend in prices after 2150. For larger differences in population assumptions, the impact on optimal carbon tax is larger still: the 2025 tax is $65 \%$ greater under the high trajectory, relative to the low, and this ratio rises to a maximum of the high population tax reaching 2.6 times the low population tax in 2085 .

Figure 5 quantitatively confirms the theoretical intuition from section 2.2; increasing the projected population growth rate is analogous to reducing the social time discount rate. The bars on the left show that moving from the RICE to the medium population assumptions

\footnotetext{
${ }^{12}$ The rate of convergence over time is only one source of heterogeneity across regions in TFP in the RICE model: regions also differ in their starting level of TFP and in their final TFP, specified in the model as a fixed final fraction of U.S. TFP. Modifying these parameters would emphasize even more sources of uncertainty about future economic inequality.
} 
causes a change in the optimal carbon tax that is comparable to reducing the discount rate by half a percentage point; the bars on the right show that moving from the low to the high population path increases the tax by as much as reducing the discount rate by a full percentage point. Because the full range of discount rates considered by the U.S. government for carbon tax purposes spans only 2.5 percentage points, and because the wellknown difference between the pure time preference assumptions of Nordhaus and Stern is 1.5 percentage points, these represent large effects, covering a sizeable portion of the range of climate policy debates.

\subsection{Mechanisms: Near-term through social welfare, long-term through the economy}

Why does a larger future population imply that climate policy should be more aggressive today? There can be at least two possibilities: first, through the social welfare function, for the reasons discussed in section 2.2, a larger future population makes average costs in the future socially more important; second, through the economy, a larger future population can be expected to emit more (O'Neill et al., 2012), consuming more of an intertemporal carbon budget and leaving less for today.

Figure 6 offers a way to understand the importance of population in the social welfare function, in the spirit of the envelope theorem or Hotelling's Lemma: it shows how the optimal time path of global temperature changes as the assumed exogenous future population growth is increased. The temperature lines for lower population scenarios are above the lines for higher population scenarios ${ }^{13}$ In other words, it is optimal to allow the world to grow hotter and to permit climate change to be more damaging if we assume there will be fewer future people to be hurt by climate damages. Moreover, the effect of this reduced "price" of

\footnotetext{
${ }^{13}$ Note that none of these scenarios keep the temperature change under the 2 degree or 1.5 degree targets highlighted by some international policy-makers, as these would require very rapid mitigation regardless of population or TFP convergence.
} 
climate change is so large as to outweigh the mechanically increased carbon "budget" of a smaller population.

Thus, we have seen that the discounting-like role of population growth in the social welfare function is one important mechanism; it is further the case that the relative importance of population in the social welfare function to population in the economy changes over time. Figure 7 presents two ways of visualizing this change over time. The top panel (a) concentrates on our baseline case of ethical parameters, where $\eta=1.5$ and $\rho=1.5 \%$. To produce this figure, two further optimizations of the carbon tax were computed, each with a different mixed combination of population path assumptions:

- SWF: Assuming population will follow the WPP high path in the social welfare function, but the low path in the economy.

- Economy: Assuming population will follow the WPP high path in the economy, but the low path in the SWF.

Both paths are named here after the portion of the model in which population is assumed to be high. Each mixed combination produces a fraction for each year:

$$
\operatorname{fraction}_{t}^{m i x}=\frac{\operatorname{tax}_{t}^{\text {mix }}-\operatorname{tax}_{t}^{\text {low }}}{t a x_{t}^{\text {high }}-t a x_{t}^{l o w}}
$$

where mix is either $S W F$ or Economy, as above, and tax $x_{t}$ is the optimal carbon tax in year $t$. Thus, this fraction reports the fraction of the difference in the optimal carbon tax between the high and low population paths that is covered by only changing to the high population in either the economy or the SWF.

Figure 7 plots the results. In the short run, the role of future population growth in the social welfare function is very important; future population growth does not yet matter in the economy because it has not yet been realized ${ }^{14}$ This impact reflects the social welfare

\footnotetext{
${ }^{14}$ Note that because the IAM is highly nonlinear, there is no reason to expect these fractions to add up to
} 
function judging the future to be more important, and therefore more demanding of our emission reductions today.

\subsection{Mechanisms: Allocation of the world population into regions}

The RICE model upon which our results are ultimately built splits the world into 12 regions; some other IAMs consider the world as a single representative economy. Representing climate economics as a single economy could either overstate or understand the effects of population growth, depending on the convexity or concavity of the damages, and on how climate damages are distributed throughout the world. If, for any global average of damages and population growth, damages are relatively large in the places where population growth is relatively high, then non-linear models such as ours would appropriately find a larger social cost of carbon if regionally disaggregated than if globally aggregated. This is especially important if we are interested in the future of developing countries: population growth is projected to be high throughout the 21st century in sub-Saharan Africa, where climate change is expected to be costly and where poor people are vulnerable. Similarly, large and growing populations are exposed to climate damages in developing countries in Asia. Population growth is low in the northern areas of Europe and North America where climate change may be less harmful.

Figure 8 compares the optimal carbon taxes resulting from our population projections with optimal carbon taxes from alternative projections in which the world total population is the same as in the projections, but each region's population is, by hypothesis, set to grow at the same global rate. Thus, for each of the WPP projections (low, medium, high), an equal growth projection $\left(\mathrm{low}^{e}\right.$, medium $^{e}$, high $\left.^{e}\right)$ is created in which population growth within time periods is equal across regions. Therefore, substituting these equal growth projections for the original WPP projections preserves any consequences of the total future world population, one in any particular year. 
while eliminating any consequences of unequal future population growth rates across regions. In particular, figure 8 plots the ratio in each year of the optimal tax with the WPP projections to the optimal tax with the equal growth projections. The fact that these ratios are greater than one indicates that the distribution of population growth across regions raises the nearterm social cost of carbon, relative to a world in which it were not the case that population growth would be particularly concentrated in developing countries.

\section{Optimal tax and TFP convergence}

Widely used computations of an optimal carbon tax assume a large decrease in future country-level global inequality, driven by rapid global convergence in Total Factor Productivity. Such convergence may not occur, especially in TFP. This section considers optimal carbon taxes under the assumption that future TFP convergence is slow. One argument

against substantial spending to avoid climate change is that its future victims will be much richer than today's world population; if future TFP convergence is much slower, this may not be true.

\subsection{Result: Slower convergence increases near-term taxes, de- creases long-term taxes}

Figure 9 presents the results of alternative assumptions for TFP convergence, which has different implications over the course of the 21st century. In the near term, as panel (a) shows by comparing the ratio of the social cost of carbon under alternative TFP assumptions to the results under the standard RICE convergence rate, slower assumed TFP convergence translates into higher optimal carbon taxes. This is because the future people in developing countries who will be harmed by climate change will be poorer than otherwise if TFP convergence is slower, so their losses are more socially costly. As the 21st century progresses, 
this pattern reverses, and each path crosses 1: higher TFP convergence comes to emphasize higher optimal carbon tax, because a richer world is able to afford more climate mitigation.

Panel (b) compares the magnitude of the consequences for the optimal 2015 carbon tax

of changing TFP convergence assumptions with the consequence of reducing the rate of pure time discounting. Each of our four TFP assumptions is computed with a lower and higher discount rate. Comparing the optimal tax with zero convergence with the optimal tax under the original RICE convergence speed, we find that eliminating TFP convergence increases the optimal tax by a magnitude about as large as decreasing the rate of pure time discounting by 0.5. This computational result is consistent with Gollier s (2015) theoretical observation that economic convergence has consequences for the valuation of future losses that are similar to increasing the rate of pure time preference.

\subsection{Interaction: TFP convergence and population growth}

We have seen that faster future population growth, especially as it is expected to be concentrated in the developing world, and slower future TFP convergence both would raise today's optimal carbon taxes. This section asks how these predictions about the future of the developing world interact — by which we mean, does the effect of changing population growth or TFP assumptions depend upon the level of the other input? This would be an especially important question to ask if economic growth and fertility reduction were not independent, but instead were causally linked to one another, as many demographers and economists have suggested (Foster et al., 2006; Vogl, 2015).

Figure 10 presents sixteen combinations of the assumptions that we have considered: population growth that is high, medium, low, or on the RICE path, crossed with TFP convergence that is fast, slow, zero, or at the original RICE speed. The figure shows that these assumptions are not merely linearly additive, but in fact interact: the effect of reducing TFP convergence is greater when future population growth is projected to be faster, and vice 
versa. Moving from the baseline RICE assumptions to the case where the optimal carbon tax is greatest (no TFP convergence, high population growth) increases the optimal 2015 tax from $\$ 90$ to $\$ 200$. Starting from the RICE assumptions and our baseline ethical parameters of $\eta=1.5$ and $\rho=1.5 \%$, this is equivalent to reducing the rate of pure time preference, $\rho$, to something below $0.5 \%$. This is close to some of the lowest discount rates in the climate economics literature.

The highlighted diagonal row emphasizes the steep consequences for climate mitigation policy if population growth and TFP convergence are correlated across world economic development scenarios. Moving from the baseline assumptions in the 2011 version of RICE to the medium population projection update and a modestly pessimistic TFP convergence rate increases the near-term social cost of carbon by $36 \%$. The social cost of carbon is more than doubled under the combination of high population growth and no TFP convergence.

\section{Robustness}

\subsection{Separate prices by region}

The RICE (Nordhaus and Boyer, 2000) IAM, like the FUND (Anthoff and Tol, 2010) and PAGE models, computes a single, global social cost of carbon. However, Chichilnisky and Heal (1994) show that a single global price is only optimal, under a social welfare function that attends to inequality across regions, if there are lump-sum transfers across regions. This is because the rich and poor regions of the world differ considerably in their ability to pay for climate mitigation, as reflected in their marginal utility of consumption. Because the crossregion economic transfers in the actual world may be below the optimal size, this section verifies that our results are qualitatively robust to allowing the social planner to choose a time path of optimal taxes for each region of the world, rather than a single global tax. Prior papers have used FUND Anthoff, 2009) and a variant of RICE (Budolfson and Dennig, 
forthcoming) to investigate the consequences for optimal prices and climate outcomes of allowing different prices by region, rather than imposing a uniform global price.

Figure 11 presents the results for India and Africa, two large regions representative of the developing world ${ }^{15}$ Unsurprisingly, the optimal tax is greater in India, which is richer, than in Africa, which is poorer. But in both cases, our two main results are qualitatively preserved. First, optimal mitigation is greater when future population growth is projected to be greater. Second, the impact of TFP convergence changes over time: slower convergence, in which developing countries will therefore be poorer in the future, translates into higher mitigation in the near term and lower mitigation in the long term.

\subsection{Intra-region economic inequality}

In the RICE, FUND, and PAGE regionally disaggregated IAMs, each region of the world is modeled as a single representative agent; DICE (Nordhaus, 2017) is a global integrated assessment models in which the entire world's consumption is a representative agent. Therefore, none of these models consider economic inequality within world regions. However, NICE, a recent modification of RICE by Dennig et al. (2015), divides the twelve RICE regions into their economic quintiles. Using NICE, optimal mitigation policy can be computed under the assumption that future climate damages are independent of income across quintiles in a region (an assumption roughly comparable to the representative agent approach), that damages are increasingly proportional to income (hurting the rich within a region most), or that damages are inversely proportional to a income (harming the poor within a region most). As Dennig, et al. show, the social cost of carbon is highly sensitive to the future within-region economic incidence of climate damages.

In figure 12, we use NICE instead of RICE, in order to verify that our results are quali-

\footnotetext{
${ }^{15}$ Richer regions such as the U.S. or Europe would not be informative to depict because, in the utilitarian optimum represented by our social welfare function, the model recommends that they fully decarbonize quickly, as the marginal cost (to that region) of climate mitigation is greater in the developing world.
} 
tatively robust to these possible assumptions about future within-region inequality. For all three assumptions about the incidence of damages, the social cost of carbon is greater for a larger future population path, by an amount that is increasing over the near future.

\section{Conclusion: Optimal climate policy depends on the future of developing countries}

There is broad consensus that climate change will be costly to future generations, and that today's generation should make economic sacrifices to reduce carbon emissions. But how much should be sacrificed? How bad should we value future climate damages to be, relative to today's economic output? Answering these questions requires assumptions about the future of the developing world. Our paper is not intended to settle the matter of the social cost of carbon. To the contrary, we demonstrate quantitatively that choosing the best climate policy depends critically on the answers to open questions in development economics.

If today's poor economies will grow into richer countries with relatively small future populations, then fewer future people will be hurt by climate change, and they will be better able to financially sustain the damages. However, if today's poor economies remain poor, and if population growth is high in poor populations, then potentially very many future people will be exposed to climate damages, which will be deeply costly on top of their poverty. This conclusion resonates with Hallegatte et al. ss (2015) argument that the costliness of climate change for well-being will depend critically on what is achieved for economic and human development in the coming few decades.

This paper has identified a potentially important reason for possibly substantially more aggressive emission reduction policy, even using moderately large social discount rates, and even by the methods of traditional optimizing IAMs, which ignore poverty and inequality within world regions (Dennig et al., 2015), and which focus only on expected outcomes, and 
not on the risk of extreme disasters (Wagner and Weitzman, 2015). If policy-makers have good reason to believe that future population growth might be high or concentrated in poor regions, that TFP convergence will be slow or zero, or that both of these might happen, then today's emission reductions should be greater. Moreover, unlike the ethical parameters, upon which debates about climate economics have substantially focused, assumptions about TFP convergence and population projections are in principle amenable to improved economic and demographic theory and forecasting, and are therefore a reason to invest in better understanding the future of the developing world. Finally, as a policy implication, our results imply that near-term investments in accelerating human and economic development, including accelerating the demographic transition, could pay dividends in reduced necessary spending on optimal climate mitigation.

\section{References}

Anthoff, David (2009) "Optimal Global Dynamic Carbon Taxation," working paper 278, ESRI.

Anthoff, David and Richard SJ Tol (2010) "On international equity weights and national decision making on climate change," Journal of Environmental Economics and Management, Vol. 60, pp. 14-20.

Arrow, Kenneth, Maureen Cropper, Christian Gollier, Ben Groom, Geoffrey Heal, Richard Newell, William Nordhaus, Robert Pindyck, William Pizer, Paul Portney et al. (2013) "Determining benefits and costs for future generations," Science, Vol. 341, pp. 349-350.

Budolfson, Mark and Francis Dennig (forthcoming) "Optimal Climate Policy and Differential Regional Abatement," in Graciela Chichilnisky and Armon Rezai eds. Handbook on the Economics of Climate Change: Edward Elgar Press. 
Caselli, Francesco (2005) "Accounting for cross-country income differences," Handbook of Economic Growth, Vol. 1, pp. 679-741.

Chichilnisky, Graciela and Geoffrey Heal (1994) "Who should abate carbon emissions?: An international viewpoint," Economics Letters, Vol. 44, pp. 443-449.

Comin, Diego, William Easterly, and Erick Gong (2010) "Was the Wealth of Nations determined in 1000 BC?" American Economic Journal: Macroeconomics, Vol. 2, pp. 65-97.

Deaton, Angus (2013) The great escape: health, wealth, and the origins of inequality: Princeton University Press.

Dennig, F. (2014) "On the welfare economics of climate change," Ph.D. dissertation, University of Oxford, http://ora.ox.ac.uk/objects/uuid: aefca5e4-147e-428b-b7a1-176b7daa0f85.

Dennig, Francis, Mark B Budolfson, Marc Fleurbaey, Asher Siebert, and Robert H Socolow (2015) "Inequality, climate impacts on the future poor, and carbon prices," Proceedings of the National Academy of Sciences, Vol. 112, pp. 15827-15832.

Easterly, William and Ross Levine (2001) "What have we learned from a decade of empirical research on growth? It's Not Factor Accumulation: Stylized Facts and Growth Models," World Bank Economic Review, Vol. 15, pp. 177-219.

Feenstra, Robert C, Robert Inklaar, and Marcel P Timmer (2015) "The next generation of the Penn World Table," The American Economic Review, Vol. 105, pp. 3150-3182.

Foster, Andrew D, Mark R Rosenzweig et al. (2006) "Does Economic Growth Reduce Fertility? Rural India 1971-99," in India Policy Forum, Vol. 3, pp. 179-213, Global Economy and Development Program, The Brookings Institution. 
Gerland, Patrick, Adrian E Raftery, Hana Ševčíková, Nan Li, Danan Gu, Thomas Spoorenberg, Leontine Alkema, Bailey K Fosdick, Jennifer Chunn, Nevena Lalic et al. (2014) "World population stabilization unlikely this century," Science, Vol. 346, pp. 234-237.

Gillingham, Kenneth, William D. Nordhaus, David Anthoff, Geoffrey Blanford, Valentina Bosetti, Peter Christensen, Haewon McJeon, John Reilly, and Paul Sztorc (2015) "Modeling Uncertainty in Climate Change: A Multi-Model Comparison,” working paper w21637, NBER.

Gollier, Christian (2015) "Discounting, inequality and economic convergence," Journal of Environmental Economics and Management, Vol. 69, pp. 53-61.

Greenstone, Michael and B Kelsey Jack (2015) "Envirodevonomics: A Research Agenda for an Emerging Field," Journal of Economic Literature, Vol. 53, pp. 5-42.

Grier, Kevin and Robin Grier (2007) "Only income diverges: A neoclassical anomaly," Journal of Development Economics, Vol. 84, pp. 25-45.

Hallegatte, Stephane, Mook Bangalore, Marianne Fay, Tamaro Kane, Julie Rozenberg, VogtSchilb Adrien, and Ulf Narloch (2015) Shock Waves: Managing the impacts of climate change on poverty: World Bank.

Hsieh, Chang-Tai and Peter J Klenow (2010) "Development accounting," American Economic Journal: Macroeconomics, Vol. 2, pp. 207-223.

Interagency Working Group on Social Cost of Carbon (2013) Technical Update of the Social Cost of Carbon for Regulatory Impact Analysis Under Executive Order 12866: United States Government.

Kohler, Hans-Peter (2012) "Copenhagen Consensus 2012: Challenge Paper on Population Growth," PSC Working Paper Series 12-03, University of Pennsylvania. 
Kossoy, Alexandre, Grzegorz Peszko, Klaus Oppermann, Nicolai Prytz, Noemie Klein, Kornelis Blok, Long Lam, Lindee Wong, and Bram Borkent (2015) State and trends of carbon pricing 2015: World Bank.

Kumar, Naresh and Andrew D Foster (2007) "Have CNG regulations in Delhi done their job?" Economic and Political Weekly, pp. 48-58.

Lam, David (2011) "How the world survived the population bomb: Lessons from 50 years of extraordinary demographic history," Demography, Vol. 48, pp. 1231-1262.

López, Humberto, Luis Servén et al. (2015) "Too Poor to Grow," Central Banking, Analysis, and Economic Policies Book Series, Vol. 21, pp. 309-350.

Mankiw, N Gregory (2009) "Smart taxes: An open invitation to join the Pigou club," Eastern Economic Journal, Vol. 35, pp. 14-23.

Milanovic, Branko (2011) Worlds apart: Measuring international and global inequality: Princeton University Press.

Nordhaus, WD and JG Boyer (2000) "Warming the world: the economics of the greenhouse effect," MITPress, Cambridge, MA.

Nordhaus, William D (2011) "Estimates of the social cost of carbon: background and results from the RICE-2011 model," working paper 17540, National Bureau of Economic Research.

_ (2017) "Revisiting the social cost of carbon," Proceedings of the National Academy of Sciences, p. 201609244.

O'Neill, Brian C, Brant Liddle, Leiwen Jiang, Kirk R Smith, Shonali Pachauri, Michael Dalton, and Regina Fuchs (2012) "Demographic change and carbon dioxide emissions," The Lancet, Vol. 380, pp. 157-164. 
Pindyck, Robert S (2013) "Climate Change Policy: What Do the Models Tell Us?" Journal of Economic Literature, Vol. 51, pp. 860-872.

Pizer, William, Matthew Adler, Joseph Aldy, David Anthoff, Maureen Cropper, Kenneth Gillingham, Michael Greenstone, Brian Murray, Richard Newell, Richard Richels et al. (2014) "Using and improving the social cost of carbon," Science, Vol. 346, pp. 1189-1190.

Pritchett, Lant (1997) "Divergence, big time," The Journal of Economic Perspectives, Vol. 11, pp. 3-17.

Ravallion, Martin (2012) "Why don't we see poverty convergence?" The American Economic Review, Vol. 102, pp. 504-523.

Scovronick, Noah, Mark Budolfson, Francis Dennig, Marc Fleurbaey, Asher Siebert, Robert Socolow, Dean Spears, and Fabian Wagner (2017) "Impact of population growth and population ethics on climate change mitigation policy," working paper, Princeton University Climate Futures Intiative.

Spears, Dean (2015) "Smaller human population in 2100 could importantly reduce the risk of climate catastrophe," Proceedings of the National Academy of Sciences, Vol. 112, pp. E2270-E2270.

Stern, Nicholas et al. (2006) Stern Review: The economics of climate change, Vol. 30: HM Treasury.

Tanaka, Shinsuke (2015) "Environmental regulations on air pollution in China and their impact on infant mortality," Journal of health economics, Vol. 42, pp. 90-103.

The National Academies (2016) Assessment of Approaches to Updating the Social Cost of Carbon: National Academies Press. 


\section{(2017) Valuing Climate Damages: Updating Estimation of the Social Cost of Carbon}

Dioxide: National Academies Press.

Vogl, Tom S (2015) "Differential Fertility, Human Capital, and Development," The Review of Economic Studies, p. rdv026.

Wagner, Gernot and Martin L Weitzman (2015) Climate shock: the economic consequences of a hotter planet: Princeton University Press.

World Bank (2010) World Development Report 2010: Development and Climate Change: World Bank. 
Figure 1: Possible future population paths: Global totals and alternative distribution across regions

(a) global population totals

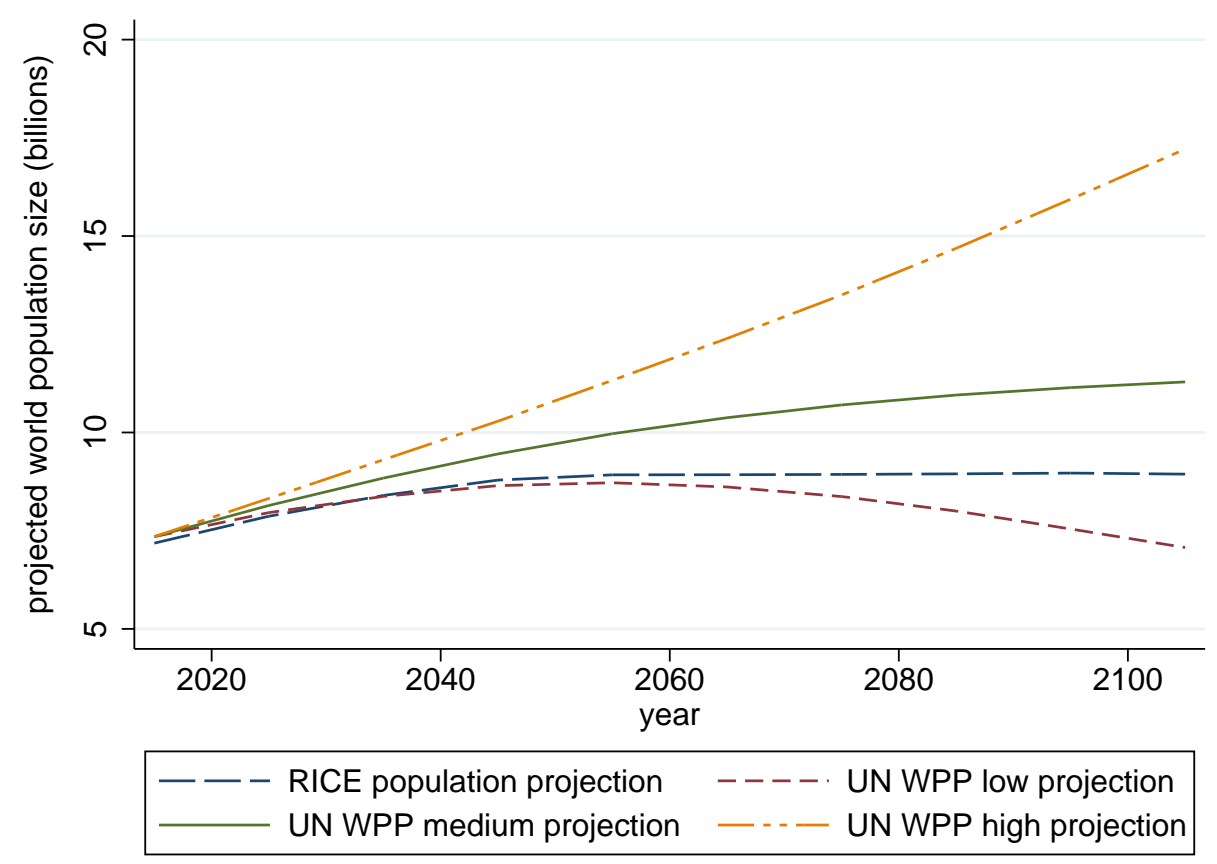

(b) decomposition of the differences between UN paths by RICE regions

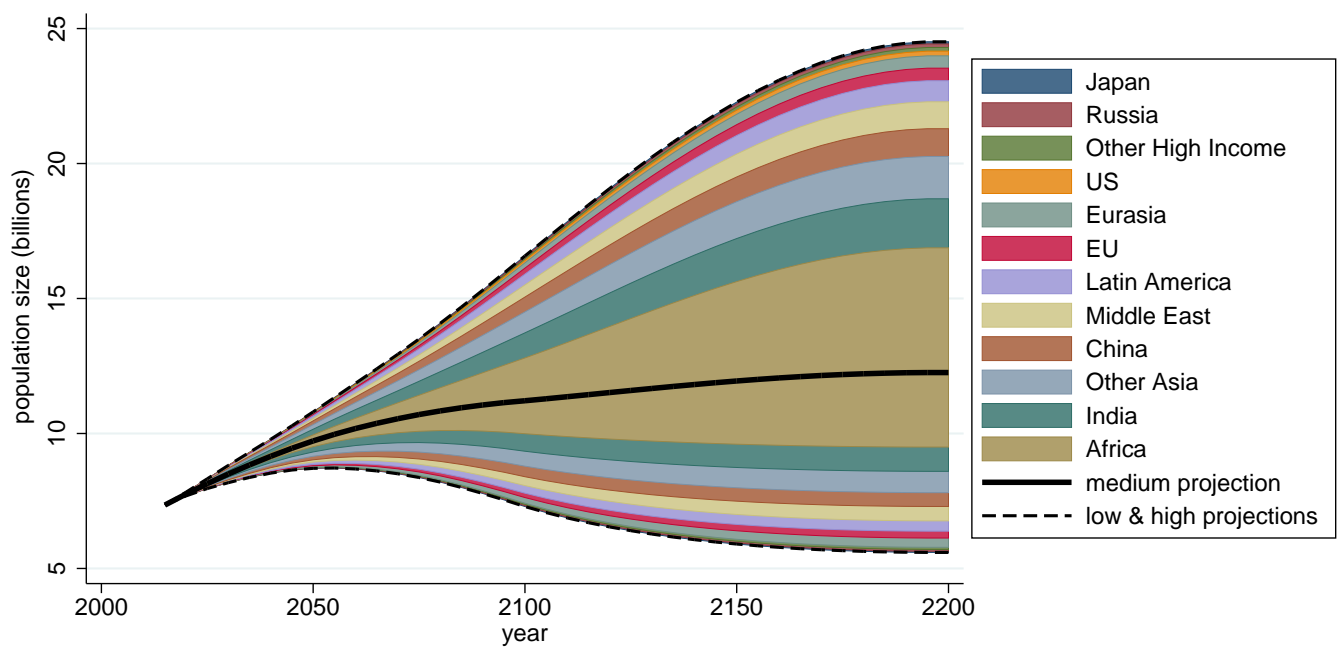

Note: We updated the 2005-2015 RICE growth rates to match observed population growth. "Middle East" refers to Middle East and North Africa; "Africa" refers to sub-Saharan Africa. 
Figure 2: Illustration: Optimal carbon price under idealized constant global population growth rates

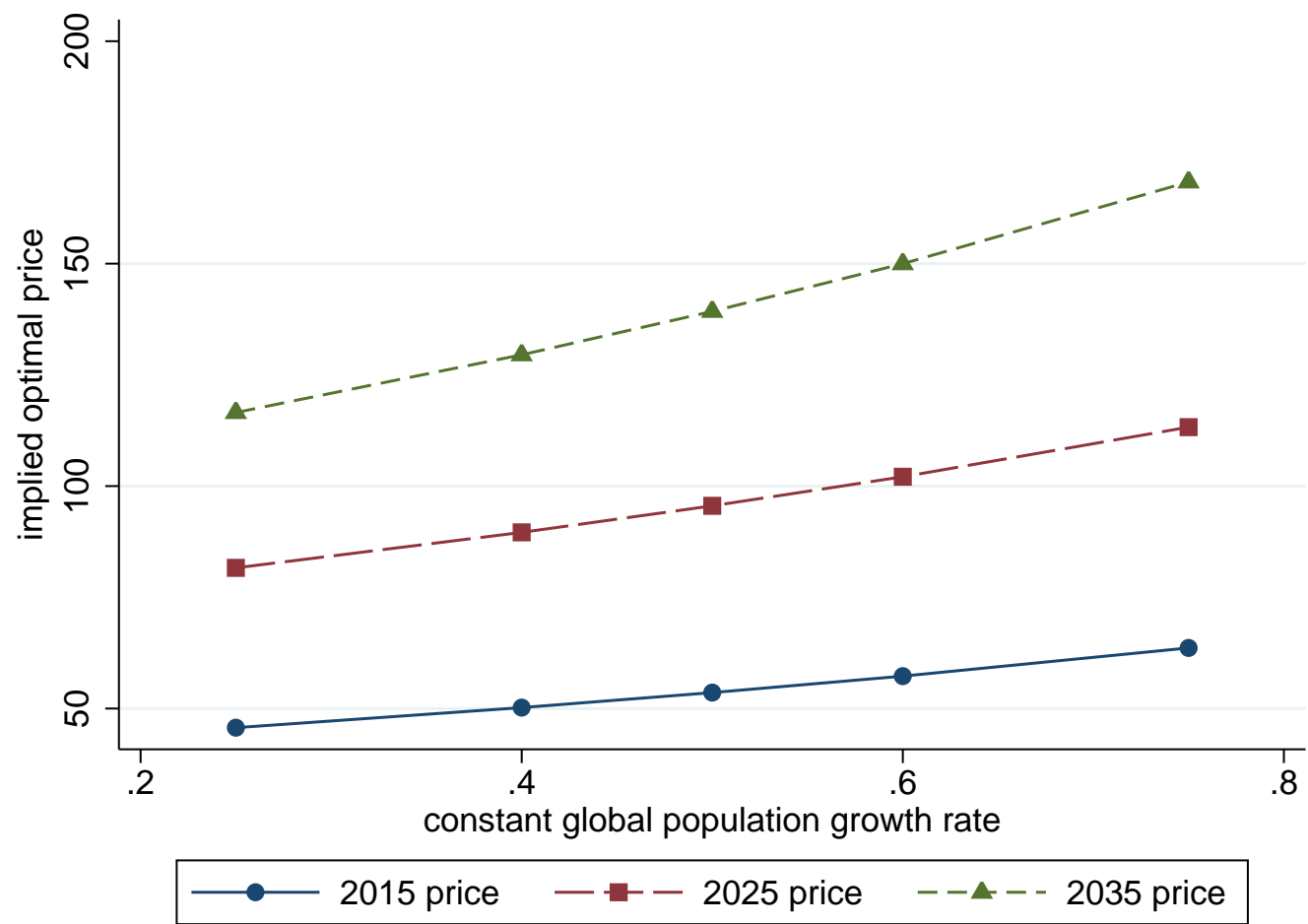

Note: $\rho=1.5 \%, \eta=1.5$. Standard RICE assumptions are used, other than for population growth. Population growth rate expressed in percentage points. Optimal price in units of $\$$ per ton C. 
Figure 3: Convergence, big time: Future within-decade Gini coefficients implied by RICE assumptions

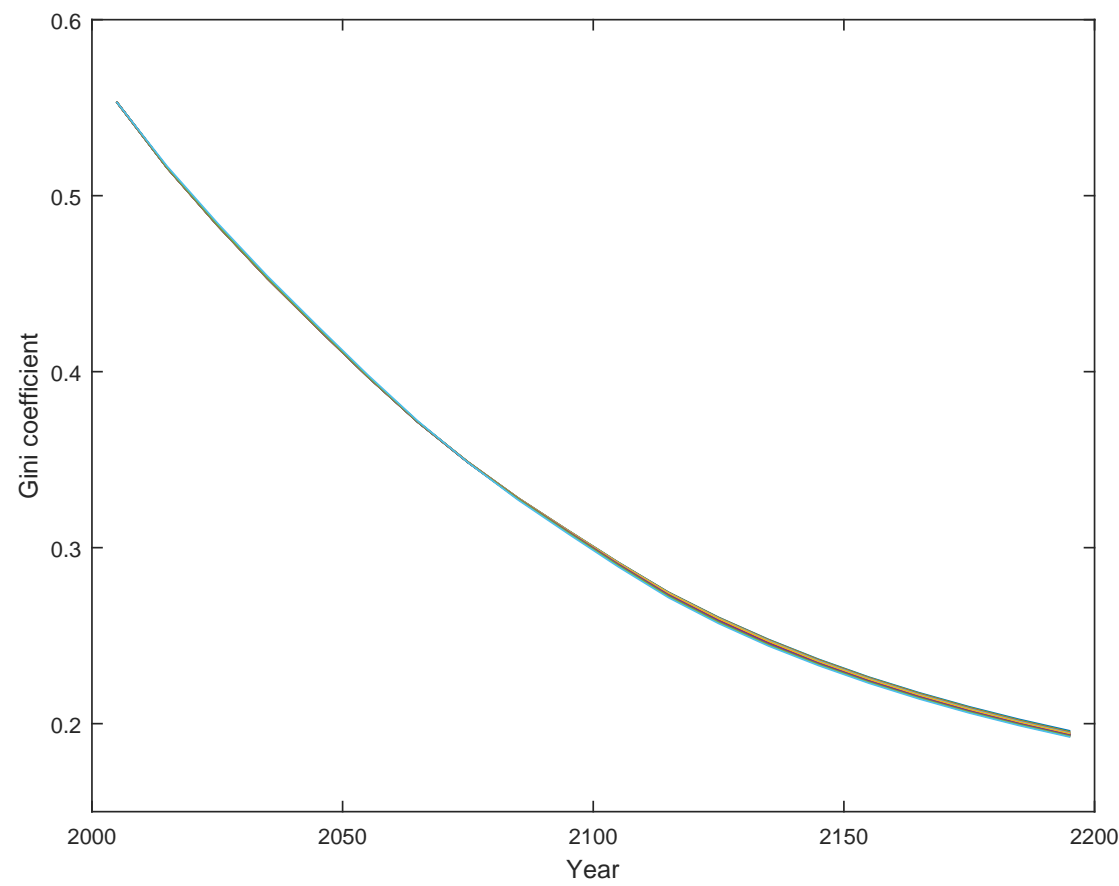

Each line is a time-path of the across-region, within-period Gini coefficients for one of 20 optimal paths yielded by RICE under 20 combinations of "ethical parameters": each combination of $\rho \in\{0,0.5,1,1.5,2\}$ in percentage points and $\eta \in\{1,1.5,2,3\}$. 
Figure 4: Result: Optimal carbon tax depends on population assumption

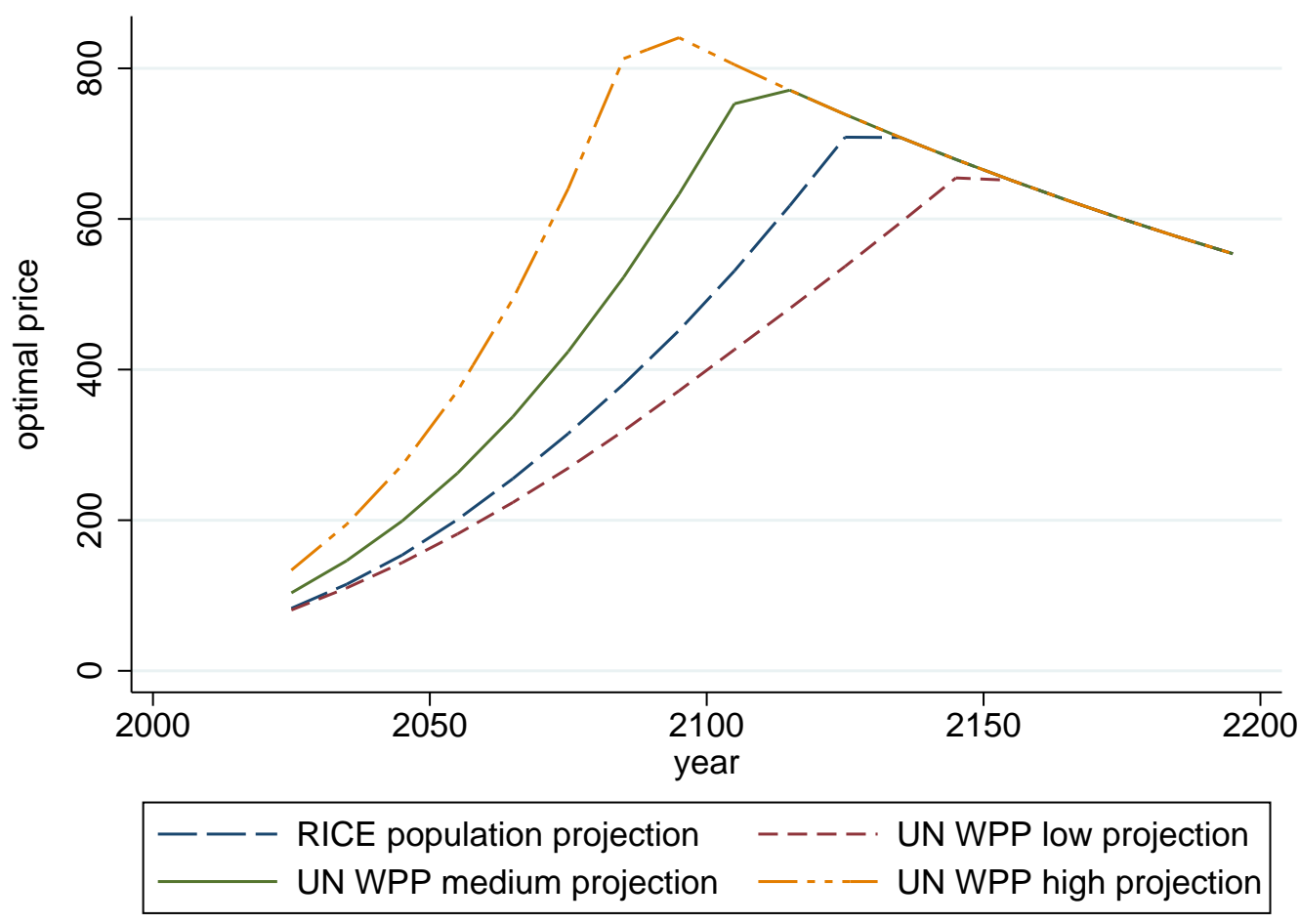

Note: $\rho=1.5 \%, \eta=1.5$. The time path of the optimal carbon tax eventually declines in all cases because the RICE model assumes that future technological progress will, over time, reduce the cost of an alternative "backstop" technology, which will eliminate an economic rationale for further net emissions. Optimal price in units of $\$$ per ton C. 
Figure 5: Result: Changes in population assumptions can have effects similar to changes in the discount rate
(a) RICE vs. medium population
(b) low vs. high population

160
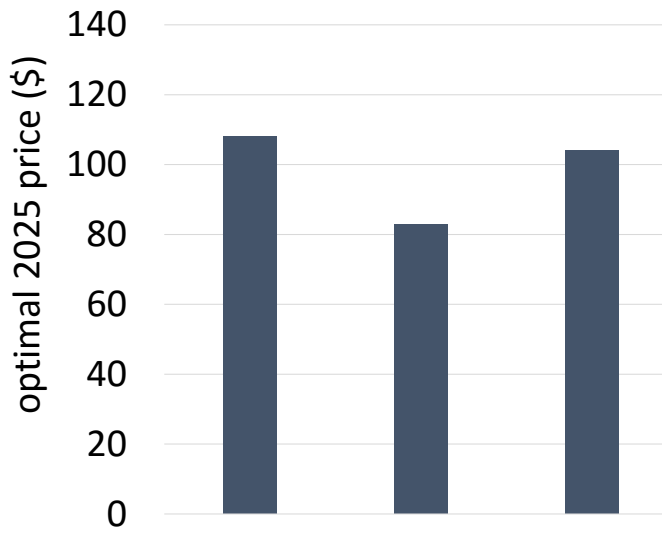

RICE pop. RICE pop. medium pop.

$\rho=1.0 \quad \rho=1.5 \quad \rho=1.5$

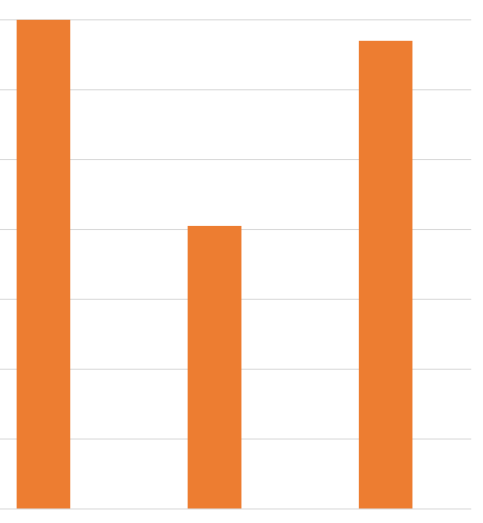

low pop. low pop. high pop.

$\rho=0.5 \quad \rho=1.5 \quad \rho=1.5$

Note: $\rho$ is expressed in terms of percentage points. Optimal price in units of $\$$ per ton $\mathrm{C}$. 
Figure 6: Mechanisms: An increased future population reduces optimal peak temperature and emissions

(a) optimal temperature path depends on exogenous population path

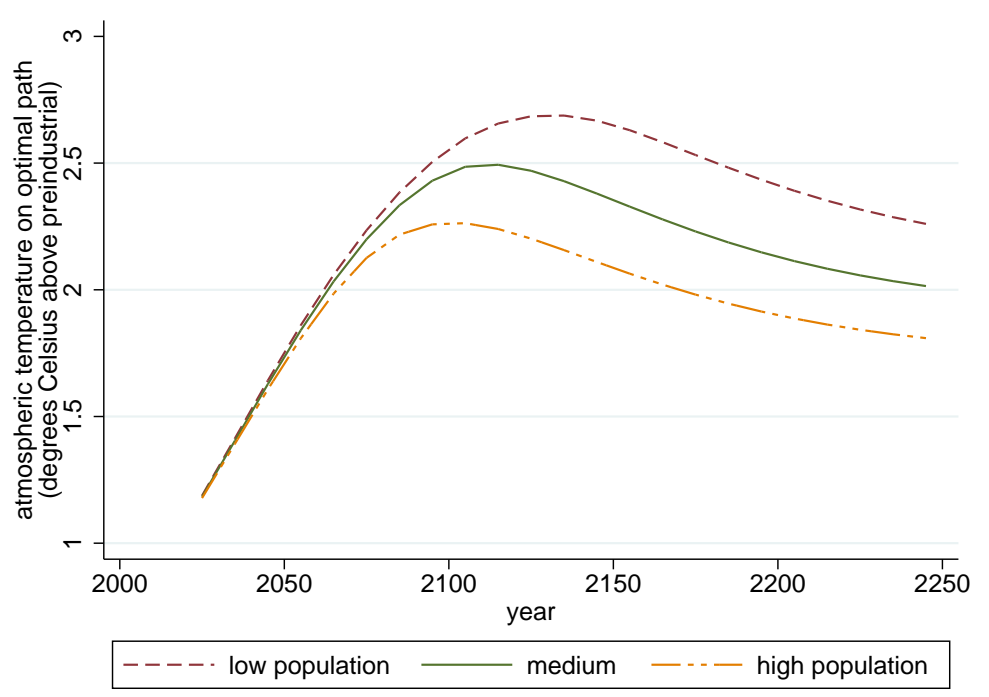

(b) optimal global emissions depend on exogenous population path

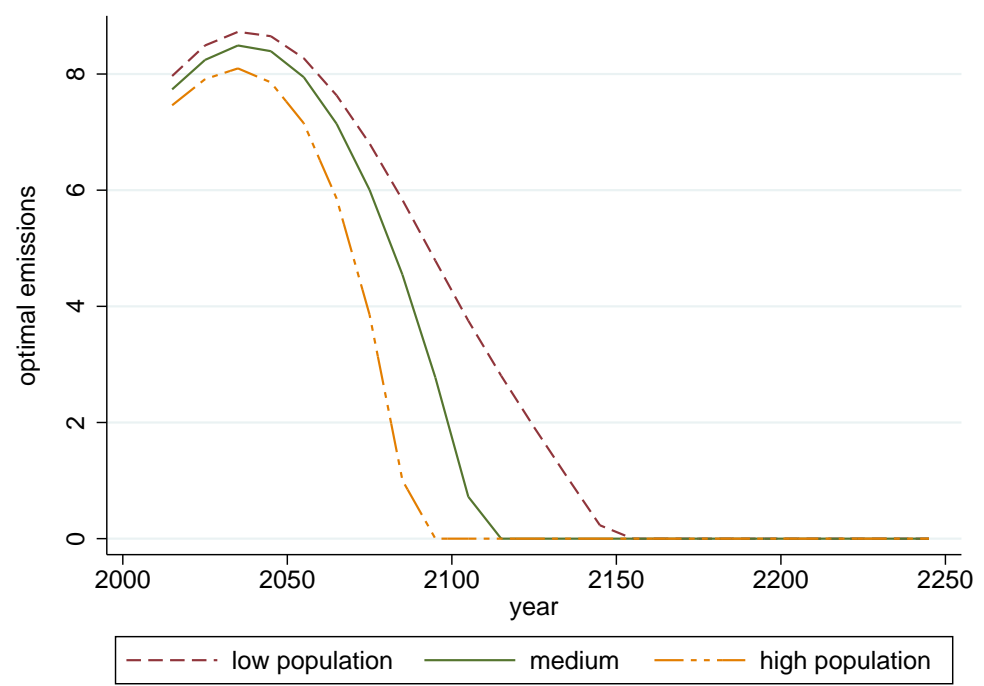

Note: $\rho=1.5 \%, \eta=1.5$. 
Figure 7: Mechanisms: Fraction of difference in optimal tax between high and low population cases accounted for by separating the roles of population in the economy and in the social welfare function

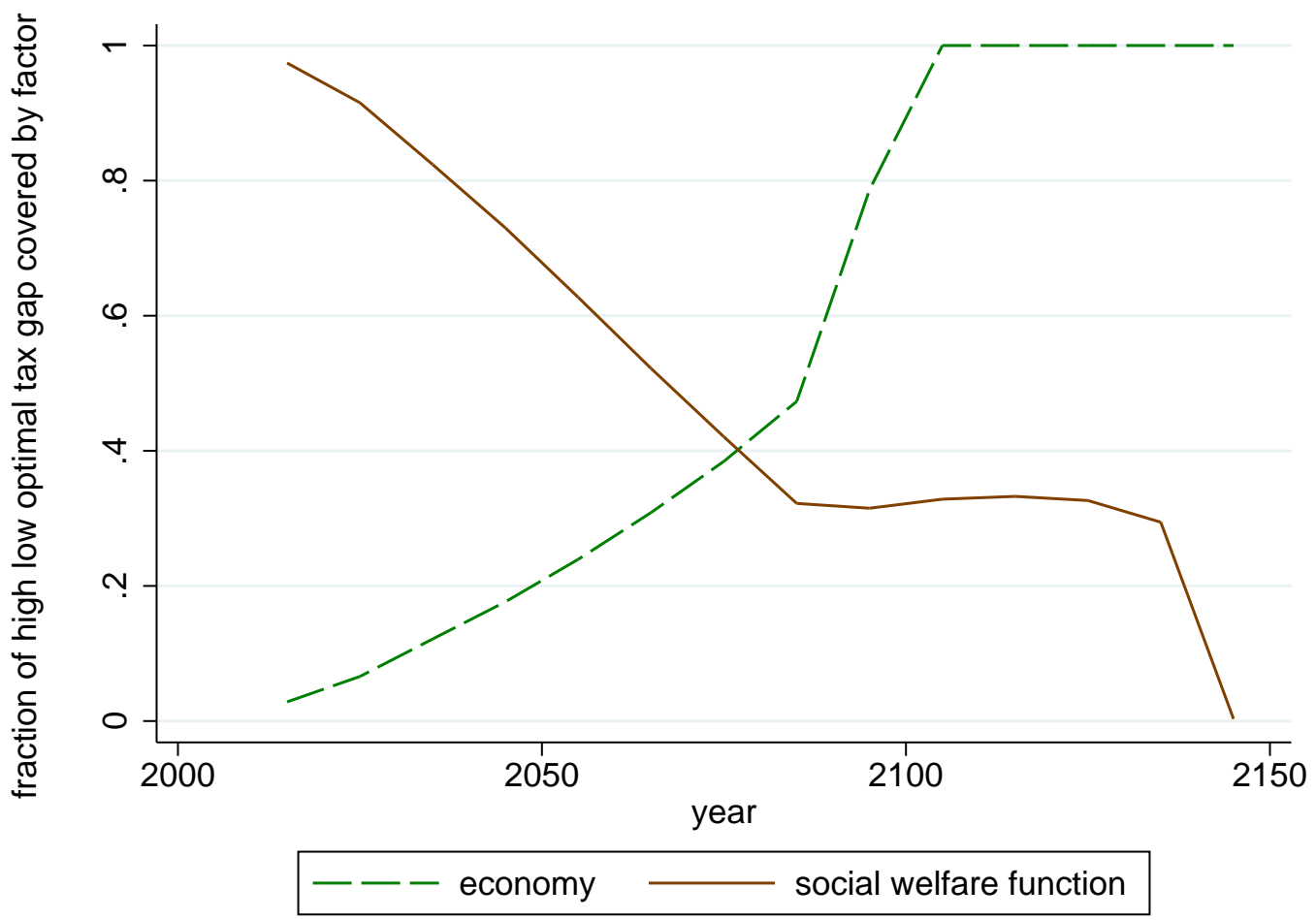

Note: For more details on the fractions plotted, see section 4.2 of the text. 
Figure 8: Mechanisms: Optimal taxes are higher than they would be in a hypothetical world in which population growth were evenly distributed across the world's regions

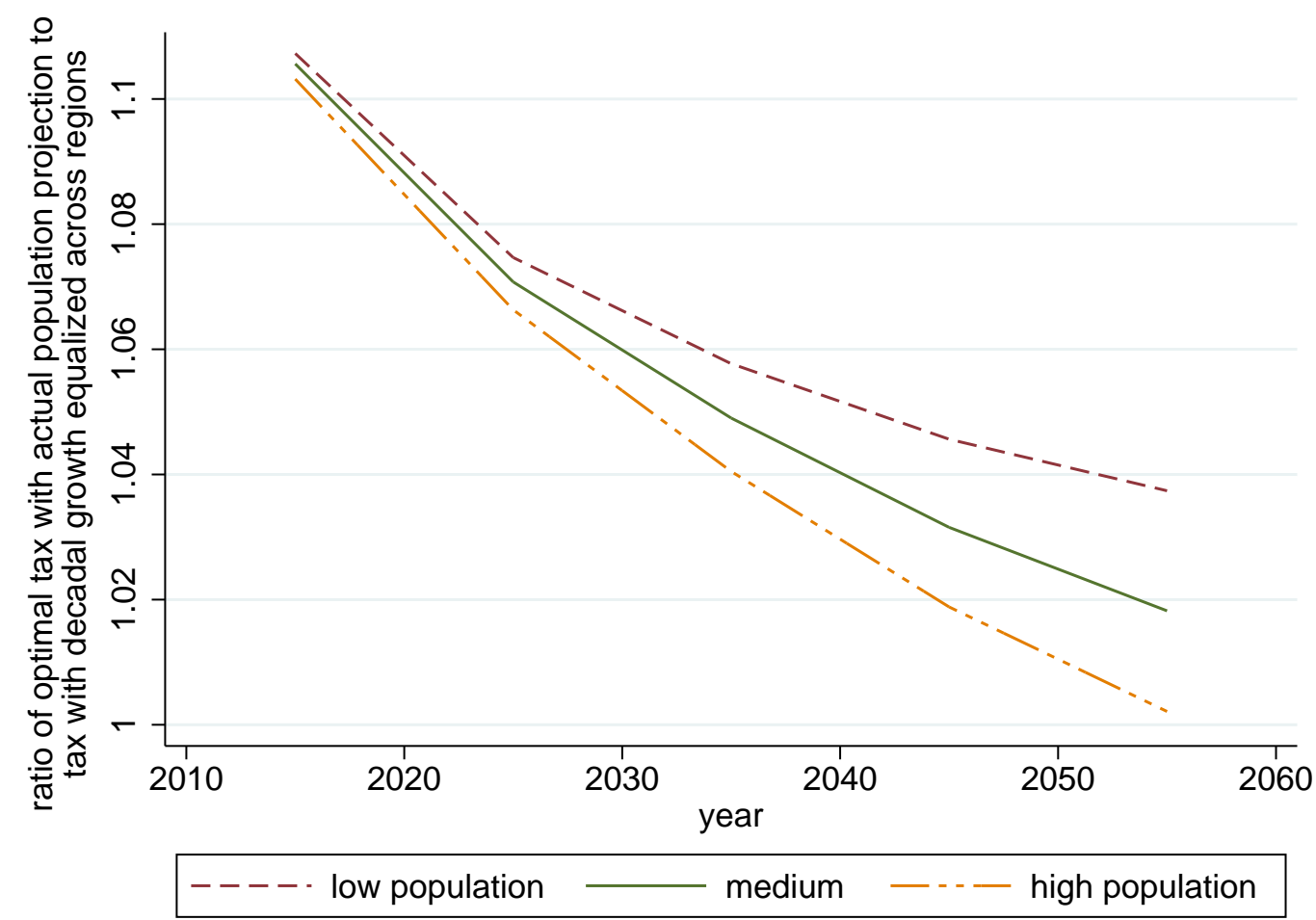

Note: For more details on the fractions plotted, see section 4.3 of the text. In both panels, the vertical axis plots the ratio of the optimal tax under UN population projections to optimal tax under hypothetical projections that preserve the future path of the world total population while assigning equal population growth rates within each time period to each region. Panel (b) presents box-and-whisker plots (showing the median and interquartile range with the box) for 20 optimization runs of the model, each with a different combination of ethical parameters $\eta$ and $\rho$. 
Figure 9: Result: Optimal carbon tax depends on assumption about future developing world TFP

(a) effects of TFP convergence are reversed as future growth and climate damages are realized $(\eta=1.5, \rho=1.5 \%)$

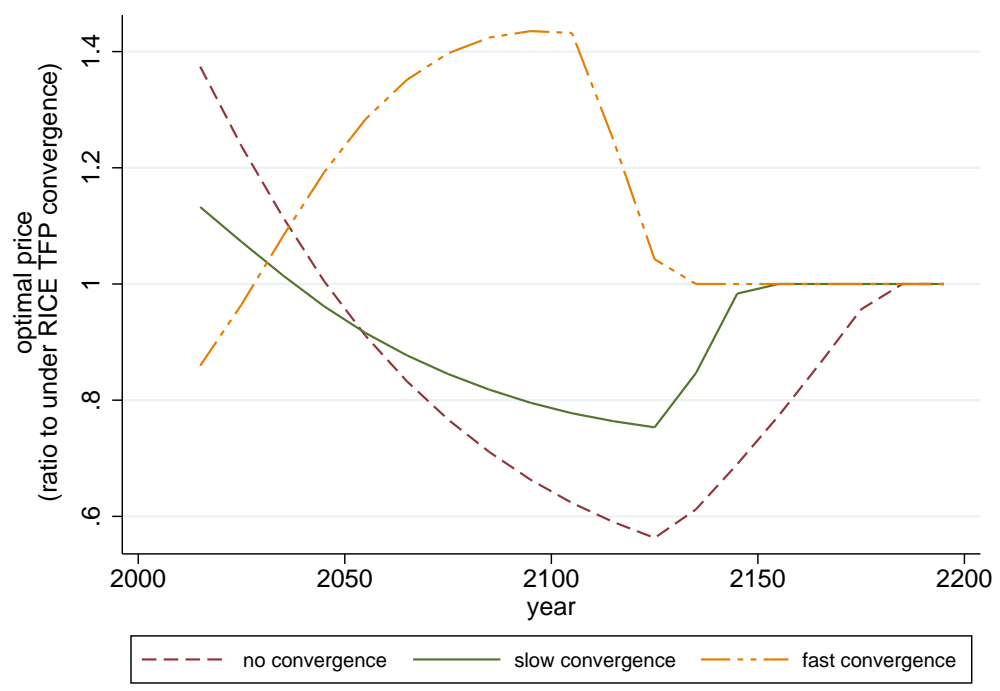

(b) reducing convergence from RICE's assumption to zero has an effect on the optimal present-day tax comparable to reducing $\rho$ by 0.5

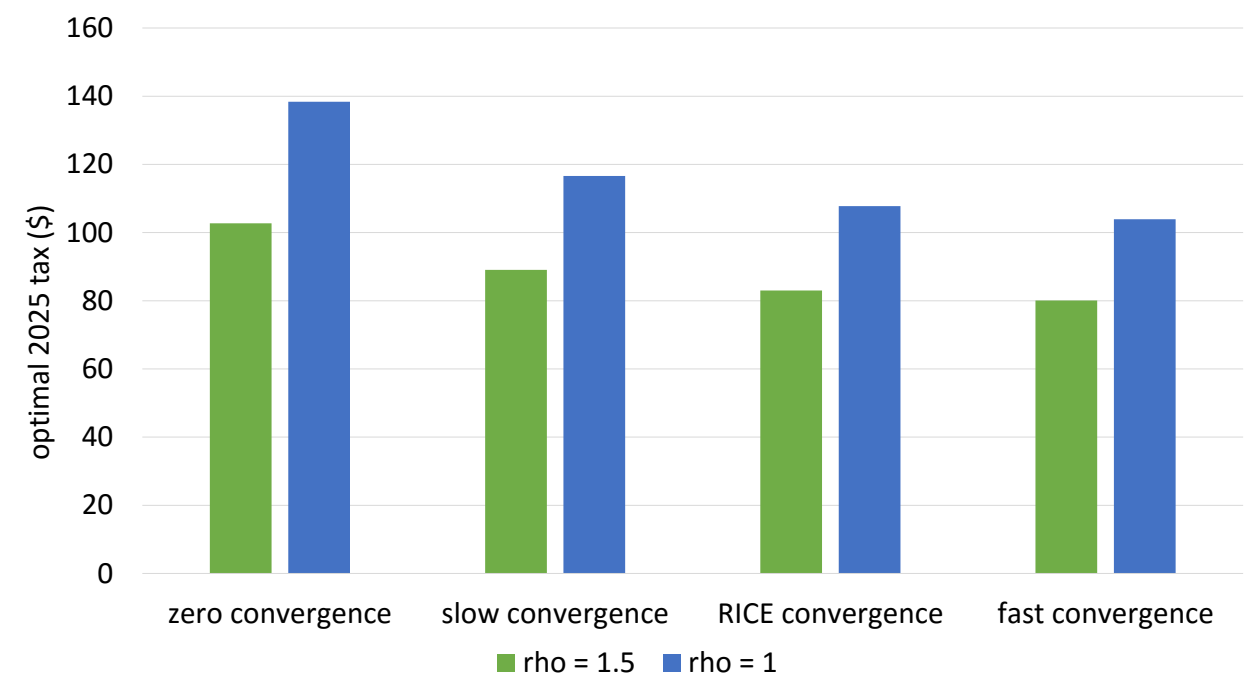

Note: All computation use the original RICE population assumptions. Panel (b) is computed using the UN WPP medium population projection and $\eta=1.5$. In panel (a), TFP assumptions eventually cease to matter (all lines go to 1 ) as all regions hit the backstop and fully decarbonize. Optimal price in units of $\$$ per ton C. 
Figure 10: Interaction: Assumptions about future population growth and TFP convergence interact

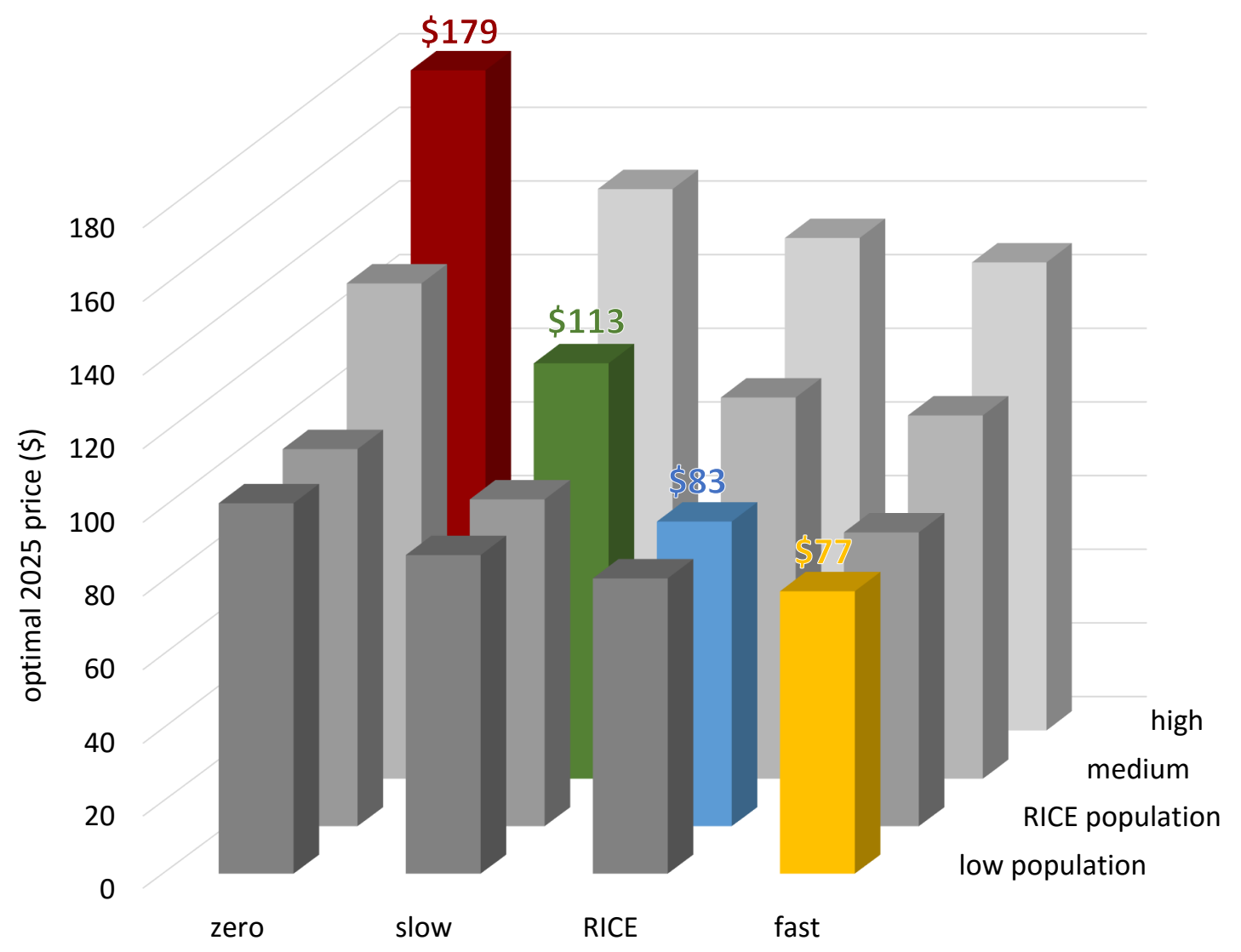

Note: $\rho=1.5 \%, \eta=1.5$. Optimal price in units of $\$$ per ton C. 
Figure 11: Robustness: Effects of population and TFP are qualitatively similar if each region is assigned a separate optimal price

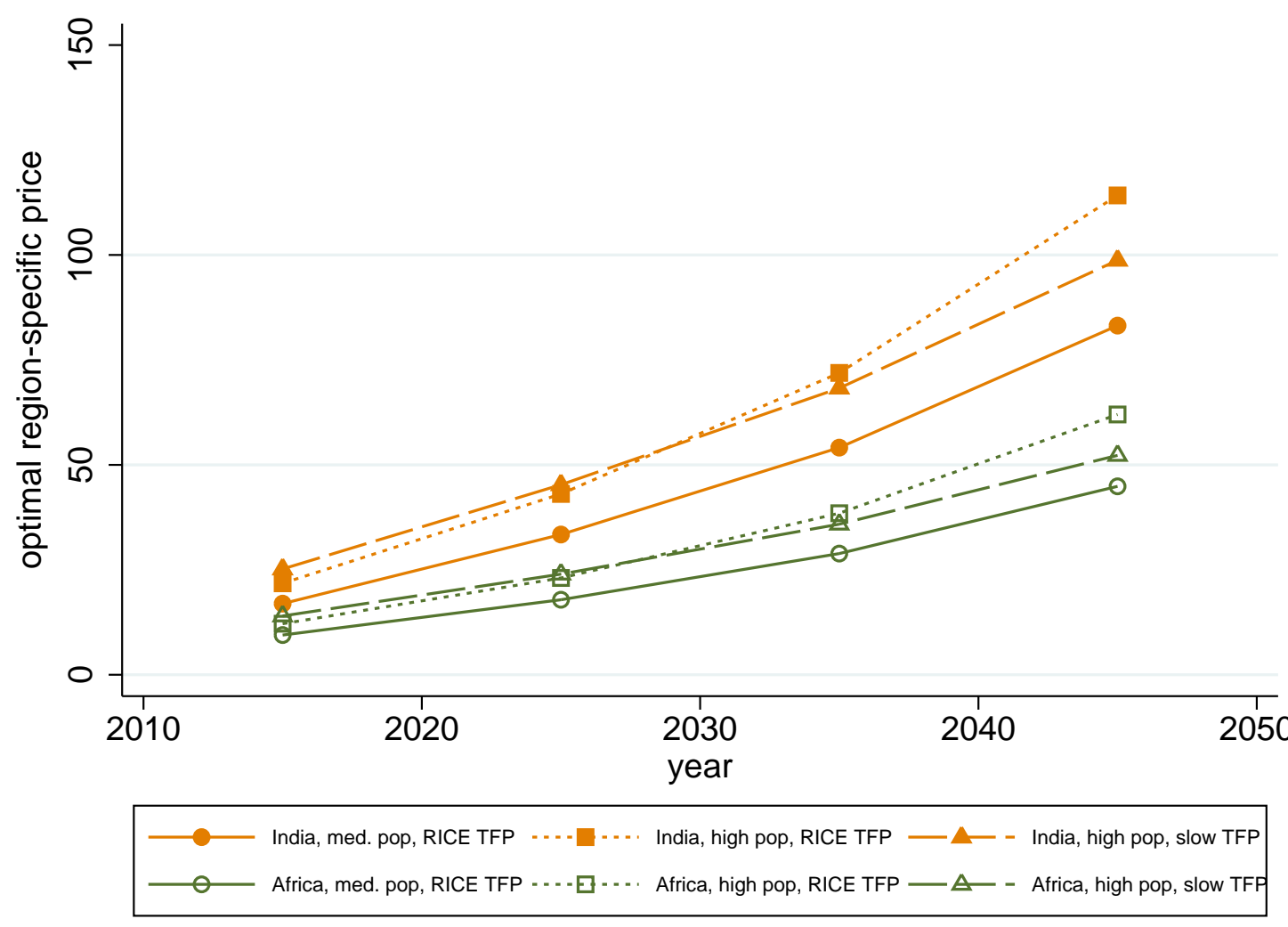

Note: $\rho=1.5 \%, \eta=1.5$. Optimal price in units of $\$$ per ton C. 
Figure 12: Robustness: Effects are qualitatively similar if damages interact with withinregion economic inequality

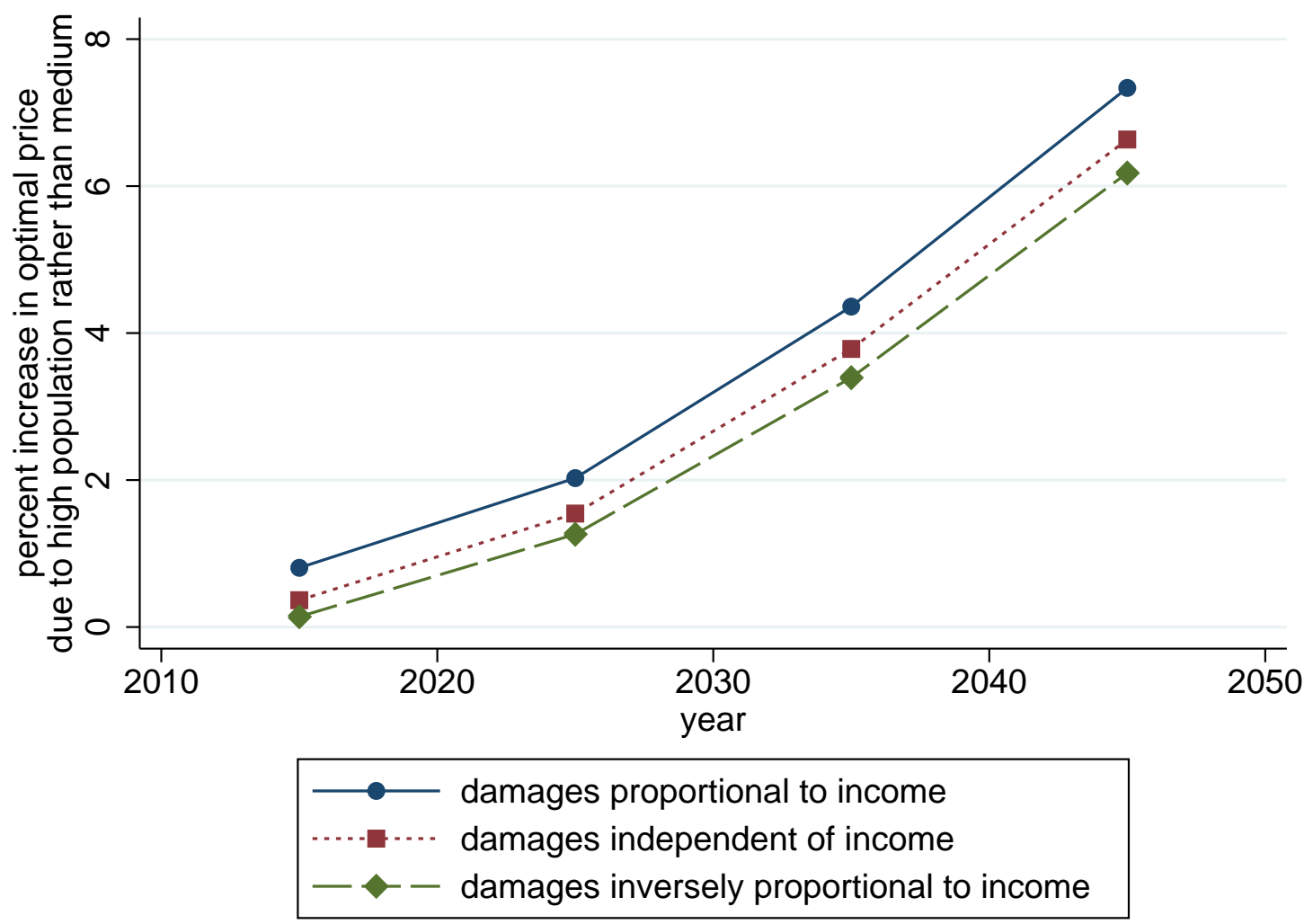

Note: $\rho=1.5 \%, \eta=1.5$. Estimated using the NICE variant of RICE, described in Dennig et al. (2015). Optimal price in units of $\$$ per ton C. 


\section{Appendix: Convergence of TFP across regions assumed by RICE}

The RICE model upon which our investigations are based assumes convergence across regions in Total Factor Productivity, which is an important determinant of convergence across regions in economic outcomes. The table below presents, for each region, the ratio of that region's TFP to TFP in the U.S.; this is done for 2015 and for the asymptotic position towards which they converge over time. The analysis in the paper varies the rate at which regions' TFP converge to their asymptotic level. Note that, for each non-U.S. region, the ratio increases from 2015 to the asymptotic value and the standard deviation across regions decreases over time.

Ratio of region-specific TFP to U.S. TFP, 2015 and asymptotically

\begin{tabular}{lrr}
\hline RICE region & TFP ratio to U.S.: asymptotically & TFP ratio to U.S.: 2015 \\
\hline U.S. & 1 & 1 \\
OECD Europe & 0.9 & 0.74 \\
Japan & 0.9 & 0.8 \\
Russia & 0.6 & 0.43 \\
non-Russia Eurasia & 0.6 & 0.24 \\
China & 0.6 & 0.2 \\
India & 0.5 & 0.13 \\
Middle East & 0.5 & 0.34 \\
Africa & 0.4 & 0.11 \\
Latin America & 0.7 & 0.33 \\
other high income & 0.9 & 0.8 \\
other non-OECD Asia & 0.6 & 0.15 \\
& & \\
standard deviation & 0.19 & 0.31 \\
\hline
\end{tabular}

